Vietnam Journal of Mechanics, VAST, Vol.41, No. 2 (2019), pp. 171-177

DOI: https://doi.org/10.15625/0866-7136/13183

\title{
ESTIMATES FOR THE ELASTIC MODULI OF 2D AGGREGATE OF HEXAGONAL-SHAPE ORTHORHOMBIC CRYSTALS WITH IN-PLANE RANDOM CRYSTALLINE ORIENTATIONS
}

\author{
Vuong Thi My Hanh ${ }^{1, *}$, Le Hoai Chau ${ }^{2}$, Vu Lam Dong ${ }^{1}$, Pham Duc Chinh ${ }^{1}$ \\ ${ }^{1}$ Institute of Mechanics, VAST, Hanoi, Vietnam \\ ${ }^{2}$ Institute of Mechanics and Environmental Engineering, Hanoi, Vietnam \\ *E-mail: vtmhanh@imech.vast.vn \\ Received: 08 October 2018 / Published online: 13 June 2019
}

\begin{abstract}
Numerical finite element simulations on the homogenization problem for large samples of particular 2D hexagonal-shape-geometry random orientation aggregates from the base crystals of orthorhombic symmetry have been performed. At sufficiently large random-aggregate samples, the scatter intervals of the macroscopic $2 \mathrm{D}$ bulk and shear elastic moduli converge toward the Voigt-Reuss-Hill bounds, and then our recently constructed theoretical estimates, which have been specified for the aggregates.
\end{abstract}

Keywords: effective elastic moduli; random cell polycrystal; 2D orthorhombic crystal.

\section{INTRODUCTION}

Polycrystalline materials often have irregular microstructures with random orientations of constituent crystals. Hence the macroscopic (effective) elastic moduli of the aggregates are expected to scatter over certain intervals depending on the sizes of the representative volume element (RVE). We still do not know if the effective moduli should converge to unique values as the size of RVE increases indefinitely or they should converge to some finite (though small) scatter intervals. Another interesting question is how the scatter intervals converge toward the known theoretical bounds constructed for the moduli [1-10] as the sizes of RVE increase, and if the bounds could be improved significantly.

In this work, we use the numerical homogenization method to compute the effective elastic moduli [11-13]. A homogenization method applies periodicity boundary condition and characteristic material force fields to obtain characteristic displacements within the representative volume. The characteristic displacement is then used to compute the effective moduli. We use the finite element method (see [14] for a review) to discretize each and every crystal grain inside of the representative volume so that we can not only

(C) 2019 Vietnam Academy of Science and Technology 
assign different properties for each grain but also solve for displacement. The periodicity boundary condition in this method gives a better accuracy compared to uniform boundary condition of physical tests or simple finite element analysis of the representative volume.

For numerical simulations and comparisons with the bounds we choose 2D polycrystals [10,15-17], which are relatively simpler than the more-complex 3D problems. In the first stage, the hexagonal-shape-geometry of the aggregates has been chosen in [10]. In [10] the crystals of the simplest square symmetry have been considered, which showed numerical scatter in the effective elastic shear modulus, while the effective elastic bulk modulus is uniquely determinable. In this paper we consider more complex orthorhombic crystals, which are expected to show the scatters in both elastic moduli.

\section{BOUNDS FOR 2D RANDOM POLYCRYSTALS}

The planar orthorhombic crystals have 4 independent elastic constants, which in Voigt notations are $C_{11}, C_{22}, C_{12}, C_{33}$. The Voigt-Reuss-Hill bounds for the effective elastic moduli of isotropic aggregates of the crystals have particular expressions

$$
K_{R} \leq K^{e f f} \leq K_{V}, \quad \mu_{R} \leq \mu^{e f f} \leq \mu_{V},
$$

where

$$
\begin{aligned}
& K_{V}=\frac{1}{4}\left(C_{11}+2 C_{12}+C_{22}\right), \quad \mu_{V}=\frac{1}{8}\left(C_{11}-2 C_{12}+C_{22}+4 C_{33}\right), \\
& K_{R}=\frac{C_{11} C_{22}-C_{12}^{2}}{C_{11}+C_{22}-2 C_{12}}, \quad \mu_{R}=2\left(\frac{C_{11}+C_{22}+2 C_{12}}{C_{11} C_{22}-C_{12}^{2}}+\frac{1}{C_{33}}\right)^{-1} .
\end{aligned}
$$

The recently constructed general bounds for the effective moduli of the random polycrystals can be well approximated by the simpler bounds for circular cell polycrystals [10,15-17], which are specified for the random orthorhombic crystal aggregate

$$
K^{L} \leq K^{e f f} \leq K^{U}, \quad \mu^{L} \leq \mu^{e f f} \leq \mu^{U},
$$

where

$$
\begin{gathered}
K^{L}=P_{K}\left(\mu_{R}, \mu_{* R}\right), \quad K^{U}=P_{K}\left(\mu_{V}, \mu_{* V}\right), \\
\mu_{* R}=\frac{K_{R} \mu_{R}}{K_{R}+2 \mu_{R}}, \quad \mu_{* V}=\frac{K_{V} \mu_{V}}{K_{V}+2 \mu_{V}}, \\
\mu^{L}=P_{\mu}\left(\mu_{R}, \mu_{* R}\right), \quad \mu^{U}=P_{\mu}\left(\mu_{V}, \mu_{* V}\right), \\
P_{K}\left(\mu_{0}, \mu_{*}\right)=\frac{\left(C_{11}+\mu_{0}+\mu_{*}\right)\left(C_{22}+\mu_{0}+\mu_{*}\right)-\left(C_{12}+\mu_{0}-\mu_{*}\right)^{2}}{C_{11}+C_{22}-2 C_{12}+4 \mu_{*}}-\mu_{0}, \\
P_{\mu}\left(\mu_{0}, \mu_{*}\right)=2\left[\frac{C_{11}+C_{22}+2 C_{12}+4 \mu_{0}}{\left(C_{11}+\mu_{0}+\mu_{*}\right)\left(C_{22}+\mu_{0}+\mu_{*}\right)-\left(C_{12}+\mu_{0}-\mu_{*}\right)^{2}}+\frac{1}{C_{33}+\mu_{*}}\right]^{-1}-\mu_{*}, \\
\mu_{*}=\frac{K_{0} \mu_{0}}{K_{0}+2 \mu_{0}} .
\end{gathered}
$$

Numerical results of the estimates (1)-(5) for some orthorhombic crystals taken from [18] are presented in Tabs. 1, 2. 
Table 1. The elastic constants $\left(C_{11}, C_{22}, C_{12}, C_{33}\right)$ of some orthorhombic crystals from [18], all in GPa

\begin{tabular}{crrrr}
\hline & $C_{11}$ & $C_{22}$ & \multicolumn{1}{c}{$C_{12}$} & $C_{33}$ \\
\hline $\mathrm{S}(1)$ & 2.05 & 4.83 & 1.59 & 0.43 \\
$\mathrm{~S}(3)$ & 2.40 & 4.83 & 1.71 & 0.87 \\
$\mathrm{U}(1)$ & 19.86 & 26.71 & 10.76 & 12.44 \\
$\mathrm{TiO}_{2}$ & 27.30 & 48.40 & 14.90 & 12.50 \\
$\mathrm{Cu}$ & 16.84 & 16.84 & 12.14 & 7.54 \\
$\mathrm{In}(2)$ & 4.45 & 4.44 & 4.05 & 0.65 \\
\hline
\end{tabular}

Table 2. The estimates (1)-(5), all in GPa

\begin{tabular}{crrrrrrrr}
\hline & \multicolumn{1}{c}{$K_{V}$} & \multicolumn{1}{c}{$K^{U}$} & \multicolumn{1}{c}{$K^{L}$} & \multicolumn{1}{c}{$K_{R}$} & \multicolumn{1}{c}{$\mu_{V}$} & \multicolumn{1}{c}{$\mu^{U}$} & \multicolumn{1}{c}{$\mu^{L}$} & \multicolumn{1}{c}{$\mu_{R}$} \\
\hline $\mathrm{S}(1)$ & 2.52 & 2.1612 & 2.1365 & 1.9900 & 0.6770 & 0.5735 & 0.5686 & 0.542 \\
$\mathrm{~S}(3)$ & 1.78 & 1.7680 & 1.7678 & 1.7600 & 0.6040 & 0.5762 & 0.5757 & 0.560 \\
$\mathrm{U}(1)$ & 17.00 & 16.7490 & 16.7400 & 16.6000 & 9.3500 & 8.5767 & 8.5544 & 8.180 \\
$\mathrm{TiO}_{2}$ & 26.40 & 24.8080 & 24.7670 & 24.0000 & 12.0000 & 11.5821 & 11.5744 & 11.400 \\
$\mathrm{Cu}$ & 14.50 & 14.4900 & 14.4900 & 14.4900 & 4.9400 & 4.0909 & 4.0279 & 3.580 \\
$\mathrm{In}(2)$ & 4.25 & 4.2475 & 4.2475 & 4.2475 & 0.4240 & 0.3579 & 0.3494 & 0.303 \\
\hline
\end{tabular}

The self-consistent approximation is the solution $K_{S C A}=K$ and $\mu_{S C A}=\mu$ of the equations

$$
K=P_{K}\left(\mu, \mu_{*}\right), \quad \mu=P_{\mu}\left(\mu, \mu_{*}\right) .
$$

\section{A 2D RANDOM MODEL AND SIMULATIONS}

The homogenization method assumes that the medium contains a large number of repeating patterns (unit cells) stacked against each other. Only one unit cell need to be analysed to compute the effective moduli. In this case, our unit cell, again, contains a large number of grains. Each grain is assigned with a uniform crystal property, but the property is random across the grains. In this study, the grain takes the hexagonal shape. We use the finite element method to solve the homogenization numerically, and in this case, each hexagonal grain is discretized using 64 quadrilateral finite elements.

The basis for numerical homozenization lies in the assumption that when an object undergo uniform macroscopic deformation, the microscopic deformation in each unit cell is not uniform. However, the boundary between two adjacent unit cells have to remain compativble, i.e. there should not be any cracks in the material. The deformation within a unit cell follows standart solid mechanics principals and constitutive model. These assumptions are sufficient to solve for the microscopic unit cell deformation using any numerical method. In this case the standard displacement-based finite element method is used. In addition, the plane-strain formulation is used for this two-dimensional problem and don't anticipate that the conclusion is different if we use plane-stress. 
To arrive at the effective moduli, we first apply the periodicity condition [13] and 3 load cases of material forces (in 3D, it would requires 6 load cases) to the unit cell. The material forces are calculated according to the following formula

$$
f^{(i j)}=-\operatorname{div}_{Y} \mathbf{C}\left[\mathbf{e}^{(k l)}\right] .
$$

After solving the unit cell for characteristic displacements, the homogenized elasticity tensor is calculated according to the following formula.

$$
C_{i j k l}^{e f f}=\frac{1}{|Y|} \int_{Y}\left(\mathbf{e}^{(i j)}+\nabla_{y} \chi^{(i j)}\right) \mathbf{C}(y)\left(\mathbf{e}^{(k l)}+\nabla_{y} \chi^{(k l)}\right) d y,
$$

where $|Y|$ is the unit cell size (the area of the unit cell in this 2D setting); $\mathbf{e}^{(i j)}$ is the unit test strain; $C_{i j k l}^{e f f}$ is the component of the homogenized elasticity tensor (to be calculated); $\mathbf{C}(y)$ is the given fourth order local elasticity tensor that varies according to the location in the unit cell; $\chi^{i j}$ is the so-called characteristic displacement corresponding to the unit test strain $\mathbf{e}^{(i j)}$.

\section{NUMERIAL RESULTS AND COMPARISONS}

The effective moduli of of some polycrystalline aggregates taken from [18] and reported in Tab. 1 with random crystal orientations are calculated using numerical homogenization. To represent the material microstructure, we assume periodic unit cells each of which contains a large number of hexagonal grains with a random crystal orientation. Each grain is described by $8 \times 8$ rectangular finite elements.

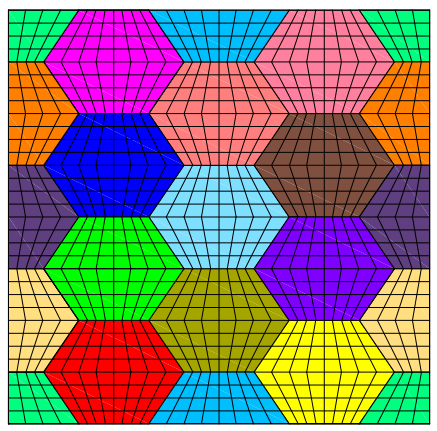

(a)

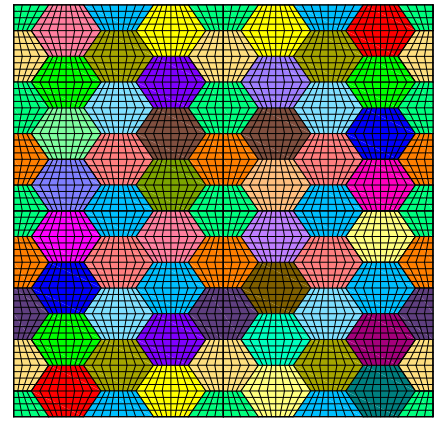

(b)

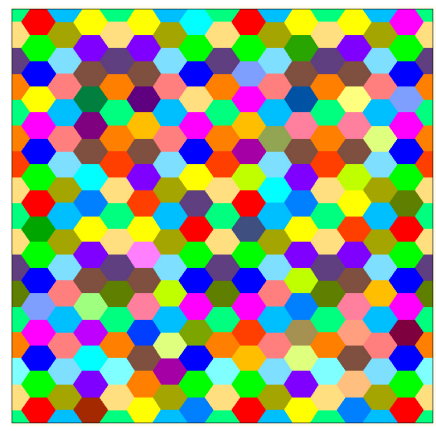

(c)

Fig. 1. Unit cell finite element meshes with $4 \times 4,8 \times 8,16 \times 16$ hexagonal grains per unit cell (a)(c); Note that colors are only used to distinguish the grains and do not correspond to orientation. Grains with the same colors can have different orientation

Five levels of grain refinements are considered, corresponding to roughly $4 \times 4,8 \times$ $8,16 \times 16,32 \times 32$, and $64 \times 64$ hexagonal grains per unit cell (see Fig. 1 for illustrations of thee finite element meshes of the unit cell at the first 3 grain sizes). The grains are assigned with random crystal orientations. The randomness in the angles between the 
crystal axes and the Cartesian coordinates is uniformly distributed between 0 and 180 degrees. For each grain size, a number of random samples are generated and analysed, each of which is represented by a marker point on the plot, c.f. the following figures.

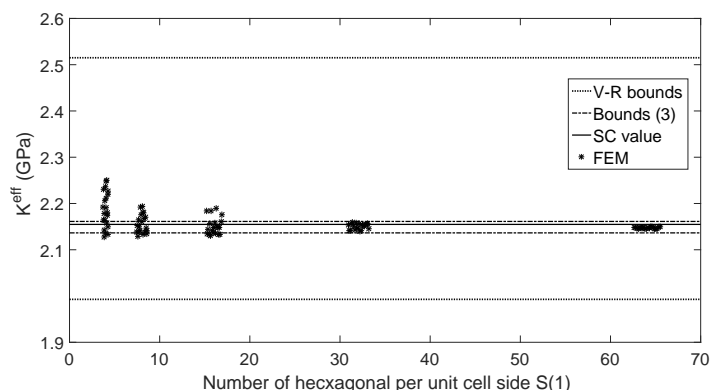

(a)

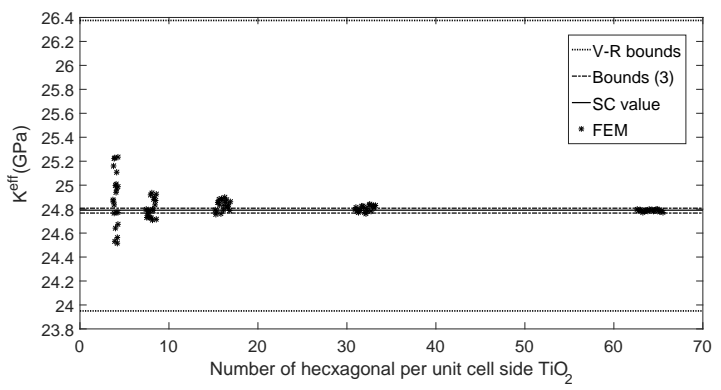

(c)

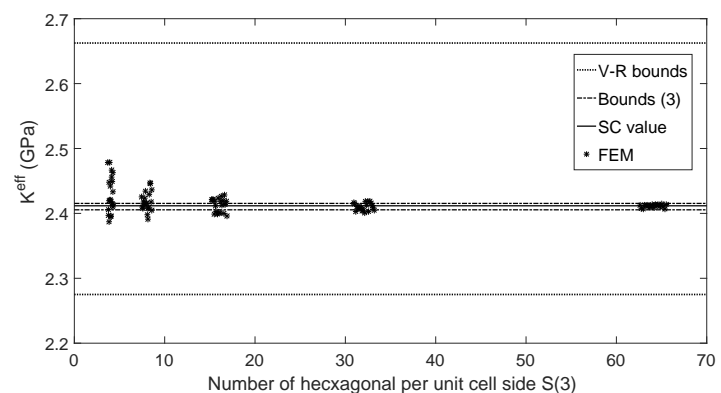

(b)

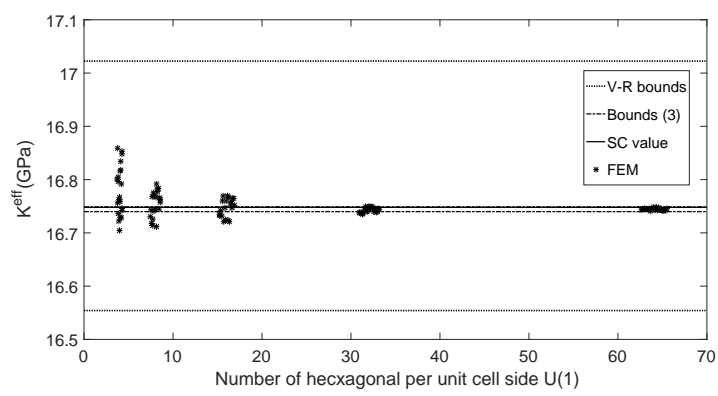

(d)

Fig. 2. The scatter of the effective $2 \mathrm{D}$ bulk moduli (in GPa) of $\mathrm{S}(1), \mathrm{S}(3), \mathrm{TiO}_{2}$ and $\mathrm{U}(1)$ aggregate with hexagonal grain configuration and random crystal orientations versus the unit cell size (in the number of grains). There are five clusters of points corresponding to the five meshes in Fig. 1 (because of close clustering of the scatter points, the horizontal positions of points are slightly shifted for plotting clarity). Finite element simulation results (FEM) are compared with VoigtReuss-Hill (V-R) bound from Eq. (1), our theoretical bounds (3), and self-consistent (SC) value from (6)

The finite element simulation results on the effective moduli compared with the bounds (1)-(5) are reported in Figs. 2-3. Maximal capacity of our computer has been used to generate the results. A computer, with operating system windows 10 Pro 64bit, hardware includes processor Intel (R) Core (TM) i5-7400 CPU 3.00GHz(4CPUs) 3.00 GHz, memory RAM 8Gb DDR3, solid state drive 120Gb SSD, has been used. Computing time for each figure is about 18 hours. One can see that the scatters of the simulation results reduce as the sizes of RVEs increase. The numerical simulation results converge toward Voigt-Reuss-Hill bounds (1), and then to the estimates (3), for both the macroscopic $2 \mathrm{D}$ bulk and shear moduli. 


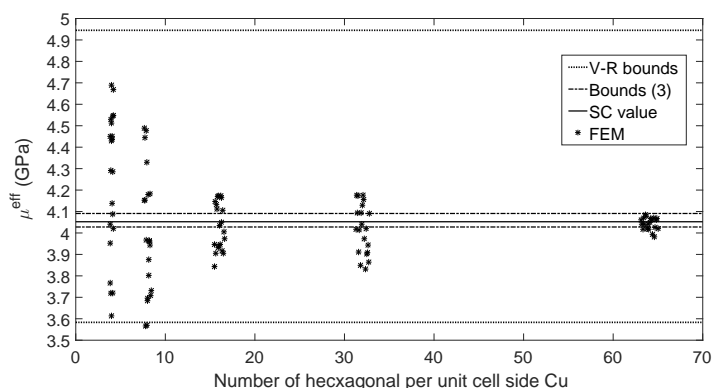

(a)

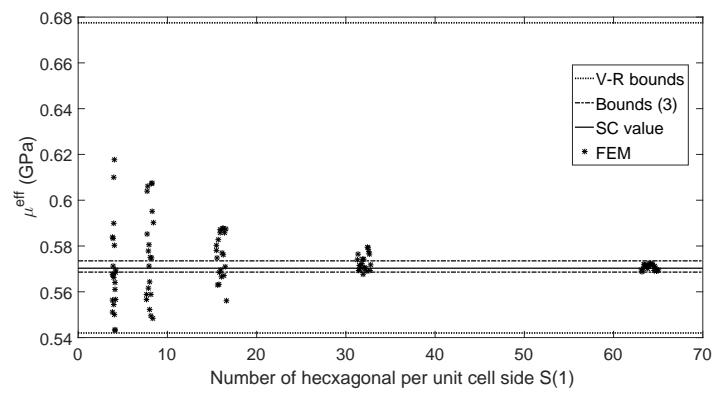

(c)

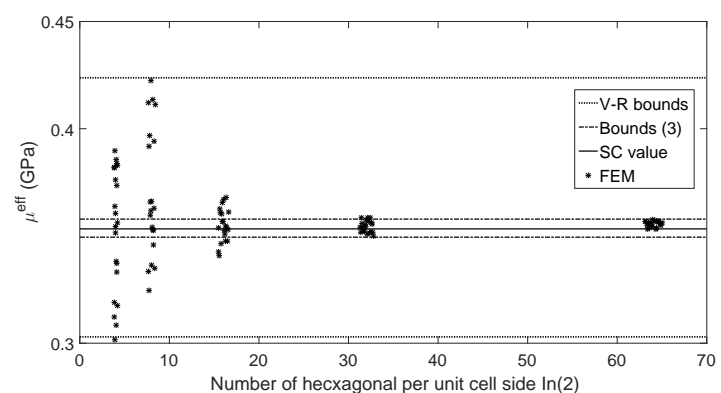

(b)

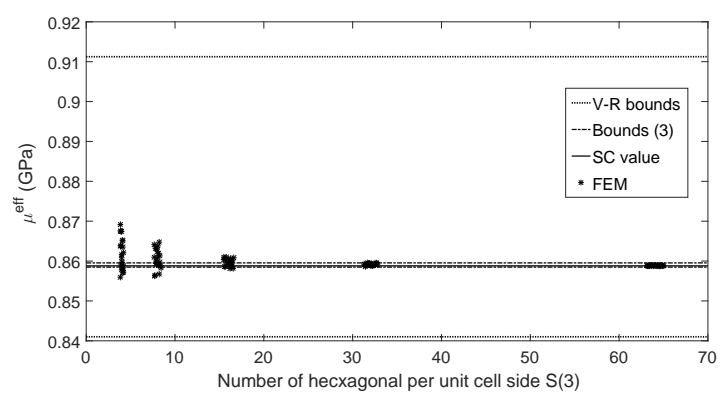

(d)

Fig. 3. The scatter of the effective shear moduli (in GPa) of $\mathrm{Cu}, \operatorname{In}(2), \mathrm{S}(1)$ and $\mathrm{S}(3)$ aggregate with hexagonal grain configuration and random crystal orientations versus the unit cell size (in the number of grains). There are five clusters of points corresponding to the five meshes in Fig. 1 (because of close clustering of the scatter points, the horizontal positions of points are slightly shifted for plotting clarity). Finite element simulation results (FEM) are compared with VoigtReuss-Hill (V-R) bound from Eq. (1), our theoretical bounds (3), and self-consistent (SC) value from (6)

\section{CONCLUSION}

At the maximal RVE size of $64 \times 64$ grains, the numerical results on both the effective bulk and shear moduli of certain hexagonal-shape-crystal aggregates with random crystalline orientations converge to our theoretical bounds on the moduli. Some other crystalline aggregates would require larger size RVEs to achieve that convergence. Still, this is a good bench mark for further studies on more complex random Voronoi polycrystals, which are more realistic and might require stronger mathematical methods and computer resources, as both the grains' shapes and crystalline orientations are random. That is the subject of our further studies.

\section{ACKNOWLEDGMENT}

This work is funded by National Foundation for Science and Technology Development of Vietnam (NAFOSTED) under grant number 107.02-2014.33. 


\section{REFERENCES}

[1] R. Hill. The elastic behaviour of a crystalline aggregate. Proceedings of the Physical Society. Section A, 65, (5), (1952), pp. 349-354. https://doi.org/10.1088/0370-1298/65/5/307.

[2] Z. Hashin and S. Shtrikman. A variational approach to the theory of the elastic behaviour of polycrystals. Journal of the Mechanics and Physics of Solids, 10, (4), (1962), pp. 343-352. https://doi.org/10.1016/0022-5096(62)90005-4.

[3] J. P. Watt. Hashin-Shtrikman bounds on the effective elastic moduli of polycrystals with orthorhombic symmetry. Journal of Applied Physics, 50, (10), (1979), pp. 6290-6295. https://doi.org/10.1063/1.325768.

[4] P. D. Chinh. Elastic moduli of perfectly random polycrystalline aggregates. Philosophical Magazine A, 76, (1), (1997), pp. 31-44. https://doi.org/10.1080/01418619708209960.

[5] G. W. Milton. The theory of composites. Cambridge University Press, UK, (2001).

[6] J. G. Berryman. Bounds and self-consistent estimates for elastic constants of random polycrystals with hexagonal, trigonal, and tetragonal symmetries. Journal of the Mechanics and Physics of Solids, 53, (10), (2005), pp. 2141-2173. https://doi.org/10.1016/j.jmps.2005.05.004.

[7] P. D. Chinh. On the scatter ranges for the elastic moduli of random aggregates of general anisotropic crystals. Philosophical Magazine, 91, (4), (2011), pp. 609-627. https://doi.org/10.1080/14786435.2010.528459.

[8] P. D. Chinh. Bounds on the elastic moduli of statistically isotropic multicomponent materials and random cell polycrystals. International Journal of Solids and Structures, 49, (18), (2012), pp. 2646-2659. https://doi.org/10.1016/j.ijsolstr.2012.05.021.

[9] V. T. M. Hanh, P. D. Chinh, and V. L. Dong. Improved estimates for the effective elastic bulk modulus of random tetragonal crystal aggregates. Vietnam Journal of Mechanics, 38, (3), (2016), pp. 181-192. https://doi.org/10.15625/0866-7136/38/3/6055.

[10] D. C. Pham, C. H. Le, and T. M. H. Vuong. Estimates for the elastic moduli of ddimensional random cell polycrystals. Acta Mechanica, 227, (10), (2016), pp. 2881-2897. https://doi.org/10.1007/s00707-016-1653-y.

[11] G. Allaire. Shape optimization by the homogenization method. Springer-Verlag, New York, (2012).

[12] M. P. Bendsoe and O. Sigmund. Topology optimization: theory, methods and applications. Springer Science \& Business Media, (2003).

[13] C. H. Le. Developments in topology and shape optimization. PhD thesis, University of Illinois at Urbana-Champaign, (2010).

[14] S. J. Hollister and N. Kikuchi. A comparison of homogenization and standard mechanics analyses for periodic porous composites. Computational Mechanics, 10, (2), (1992), pp. 73-95. https://doi.org/10.1007/bf00369853.

[15] P. D. Chinh. Bounds for the effective elastic properties of completely random planar polycrystals. Journal of Elasticity, 54, (3), (1999), pp. 229-251. https://doi.org/10.1023/A:1007623720169.

[16] P. D. Chinh. Bounds on the elastic moduli of completely random two-dimensional polycrystals. Meccanica, 37, (6), (2002), pp. 503-514. https://doi.org/10.1023/A:1020943815452.

[17] D. C. Pham. Revised bounds on the elastic moduli of two-dimensional random polycrystals. Journal of Elasticity, 85, (1), (2006), pp. 1-20. https://doi.org/10.1007/s10659-006-9065-1.

[18] H. H. Landolt and R. Börnstein. Group III: Crystal and solid state physics, Vol. 11. SpringerVerlarg, (1979). 